\title{
Uusioersily
}

\section{Estimation of ocular axial length from conventional optometric measures}

Morgan, P., McCullough, S., \& Saunders, K. J. (2020). Estimation of ocular axial length from conventional optometric measures. Contact Lens and Anterior Eye, 43(1), 18-20. https://doi.org/10.1016/j.clae.2019.11.005

Link to publication record in Ulster University Research Portal

\author{
Published in: \\ Contact Lens and Anterior Eye
}

Publication Status:

Published (in print/issue): 29/02/2020

DOI:

10.1016/j.clae.2019.11.005

\section{Document Version}

Author Accepted version

\section{General rights}

Copyright for the publications made accessible via Ulster University's Research Portal is retained by the author(s) and / or other copyright owners and it is a condition of accessing these publications that users recognise and abide by the legal requirements associated with these rights.

\section{Take down policy}

The Research Portal is Ulster University's institutional repository that provides access to Ulster's research outputs. Every effort has been made to ensure that content in the Research Portal does not infringe any person's rights, or applicable UK laws. If you discover content in the Research Portal that you believe breaches copyright or violates any law, please contact pure-support@ulster.ac.uk. 


\section{Manuscript Details}

Manuscript number

Title

Article type

Keywords

Corresponding Author

Order of Authors
CLAE_2019_251_R1

Estimation of ocular axial length from conventional optometric measures Short Communication

Axial length, topography, refraction, refractive error, myopia

Philip Morgan

Philip Morgan, Sarah McCullough, Kathryn Saunders

\section{Submission Files Included in this PDF}

\section{File Name [File Type]}

Manuscript after reviewer comments without title page red line.docx [Response to Reviewers (without Author Details)]

Title page only.docx [Title Page (with Author Details)]

Response to referees.pdf [Manuscript (without Author Details)]

Figure 1.pdf [Figure]

Figure 2.pdf [Figure]

To view all the submission files, including those not included in the PDF, click on the manuscript title on your EVISE Homepage, then click 'Download zip file'.

\section{Research Data Related to this Submission}

There are no linked research data sets for this submission. The following reason is given:

The data that has been used is confidential 


\section{Introduction}

The increasing incidence of myopia across the globe [1], and in East Asia in particular [2-5], has caused great interest in the optometric community, not least because of the emergence of treatment options such as soft dual-focus design contact lenses [6,7], orthokeratology contact lenses [8-11] and pharmaceutical agents [12].

Whilst myopia can certainly be a general inconvenience, requiring the use of refractive correction usually in the form of spectacles and contact lenses, the main focus of recent concern is the pathological consequence of an eye which is fundamentally too long for its refractive capability, with higher levels of retinopathy [13], retinal detachment [14], glaucoma [15] and cataract [16] seen in myopic eyes.

In optometric circles, the degree of myopia is typically described in refractive error terminology. This is entirely logical when the primary consideration is vision correction and refraction. Myopes are described in terms of the lens power required to correct refractive error and indeed, refractive error is described as being associated with myopic pathology [17]; however, when the key clincal consideration is the pathological consequences of increased eye size (rather than referactive concerns), it seems more appropriate to describe ocular dimensions than refractive error.

Various dimensional terms are potentially available (e.g. global volume) for such a description but the most commonly used, primarily due to its relatively straightforward measurement, is axial length. Related to this, Cheng, Brennan and co-workers have recently argued that the impact of any form of myopia management is best described as its effect on eye growth rather than the slowing of refractive error change [18].

Of course, there is a close relationship between refractive error and axial length but an inspection of myopia-related pathology suggests axial length is the more important factor. In an assessment of over 9,000 patients, Tideman et al. included both axial length and refractive error in a statistical model exploring the likelihood of visual impairment [19] and reported that axial length demonstrated a significant relationship with visual impairment but refractive error did not.

Traditionally, ocular axial length was assessed using A-scan ultrasound methods but over the past 20 years, more sophisticated, non-contact, rapid instrumentation has become available. Such devices include the IOLMaster (v3, v5 and 500) (Carl Zeiss) and the newer IOLMaster 700 (Carl Zeiss) which employ partial coherence interferometry for biometric estimates, the Lenstar LS 900 (Haag-Streit) which employs low coherence reflectometry [20] and the Aladdin (Topcon) [20] which utilises a similar approach [21]. Such devices were initially developed to assist with the selection of intra-ocular lens power for patients presenting for cataract surgery. They are relatively expensive, typically costing around $£ 20,000$ to $£ 40,000$. Such a cost is justifiable in a surgical setting or in a research centre working on myopia treatment, but for optometrists and opticians interested in myopia control (especially in the early stages of this new form of refractive management) such devices have very limited use for other types of patients and as such, the cost is likely to be prohibitive. Anecdotal reports suggest that there are fewer than 20 infrared biometers in optometric practices in the United Kingdom. 
An alternative approach is to explore the potential of estimating axial length from refractive error alone or from a combination of refractive error and corneal curvature. To a first order of approximation, it seems reasonable to suppose that these three optometric measures should be associated and as refractive error estimation and corneal shape measurements are fundamental competencies of all optometrists, such analysis harbours the potential for a simple and inexpensive route to axial length measurement as an aid for eyecare practitioners wishing to consider myopia management in children.

\section{Methods}

\section{Generation of relationship}

Data from a multi-centre study of novel dual focus soft, daily disposable contact lenses were used to generate the best fit relationship between axial length versus refractive error and corneal curvature. This study has recently been reported in detail [7] but in brief, 144 subjects aged 8-12 years were examined annually for 36 months, having been fitted after a baseline assessment with a dual focus contact lens (Misight(R) 1 day, CooperVision, Inc) or a conventional design, spherical lens (Proclear(R) 1 day, CooperVision, Inc). Topography and axial length measures were evaluated at each visit with an IOL Master 500 (Carl Zeiss, Oberkochen, Germany) and cycloplegic and non-cycloplegic refractive errors were determined with a WR-5100K or WAM-5500 autorefractor device (Grand Seiko Co., Hiroshima, Japan).

Using data for all visits over thre three years of the study, aA linear mixed model was constructed to evaluate the potential for calculating the reciprocal of axial length from the reciprocal of mean anterior corneal radius of curvature and spherical equivalent refractive error at the corneal plane. Also included in the model were 'eye', nested within 'subject', which was treated as a random effect. The performance of using the regression model to calculate axial length in comparison to the measured biometer values (i.e. calculated axial length vs. measured axial length) was assessed by constructing Bland-Altman charts and by determining the $95 \%$ limits of agreement [22].

\section{Assessment with a separate dataset}

To evaluate the efficacy of the determined relationship, a comparison between measured and calculated axial length values was performed on a separate dataset. Here, values were used from the Northern Ireland Childhood Errors of Refraction (NICER) study of Saunders and colleagues [23-25]. Data were available for 1,046 young people (age six to 22 years, $99 \%$ of whom were white) on whom auto-refraction (SRW-5000 or NVision-K 5001, ShinNippon, Tokyo, Japan), anterior cornea radius of curvature and axial length determination (IOL Master v3 Carl Zeiss, Oberkochen, Germany) were assessed. Again, a Bland-Altman assessment was conducted to calculate the $95 \%$ limits of agreement.

\section{Results}

Using cycloplegic refraction data from the Chamberlain et al. study [7], the model found the following predictive relationship:$$
1 \quad 0.22273
$$$$
\frac{1}{A}=\frac{0.22273}{k}+0.00070 S+0.01368
$$ 
Where $A=$ axial length $(\mathrm{mm}), \mathrm{k}=$ mean anterior corneal radius of curvature $(\mathrm{mm})$ and $\mathrm{S}=$ spherical equivalent refractive error at the corneal plane (D). Here, both $\frac{1}{k}(F=1636, p<$ $0.0001)$ and $S(F=1334, p<0.0001)$ were significant factors, with $r^{2}=0.83$. Reorganisation of this equation to calculate axial length gives:

$$
A=\frac{1}{\frac{0.2273}{k}+0.00070 S+0.01368}
$$

Figure 1 shows the Bland Altman chart for the relationship between measured and calculated axial length. The $95 \%$ limits of agreement for the two measures are $\pm 0.73 \mathrm{~mm}( \pm 3.0 \%$ of the mean axial length measurement).

When this exercise is-was repeated for non-cycloplegic measures, the $95 \%$ confidence limits are-were $\pm 0.75 \mathrm{~mm}( \pm 3.0 \%)$. These limits of agreement were larger if only the refractive error was included in the model and corneal radius of curvature was ignored $( \pm 1.26 \mathrm{~mm}$ $[ \pm 5.1 \%]$ and $r^{2}=0.57$ for both cycloplegic and non-cycloplegic measures).

When this formula was employed for the NICER database, there was a small offset error between the two methods, with values $0.13 \mathrm{~mm}$ longer on average with the calculated values than those measured (Figure 2). The $95 \%$ limits of agreement were -0.73 to $+0.99 \mathrm{~mm}$ (an average of $\pm 3.7 \%$ ).

\section{Discussion}

Using refraction and keratometry data from the analysed dataset was able to provide reasonable predictive capability for determining absolute axial length. Incorporation of keratometry measures into the calculation offers much better agreement than refraction alone. Interestingly, similar findings were observed whether the refraction data were collected via a cycloplegic or non-cycloplegic refraction.

The limits of agreement of around $\pm 0.73 \mathrm{~mm}$ or $\pm 3 \%$ are small in absolute terms and allows for a good estimate of axial length. For example, Tideman et al. outlined the risk of visual impairment for five sub-groups of axial length: less than $24 \mathrm{~mm}, 24-26 \mathrm{~mm}, 26-28 \mathrm{~mm}, 28-$ $30 \mathrm{~mm}$ and greater than $30 \mathrm{~mm}$ [19]. The derived formula can readily assign patients to these 'risk groups' and assist practitioners in deciding whether some form of myopia management is warranted.

The predictive formula performed similarly with the data from the NICER study, with a modest offset error and $95 \%$ confidence limits of $\pm 3.7 \%$. This result is perhaps surprisingly good given the different instrumentation and protocols employed across the two studies. It would certainly be possible to modify this relationship for different clinical scenarios (e.g. different age ranges) and equipment - and certainly further work is required to understand this better - but this first overview suggests that the formula may be resilient to diverse clinical situations. 
It is important to note that whilst the predictive capability of this formula seems reasonable for absolute measures of axial length, it is unlikely to be helpful in tracking changes in axial length over time or with different treatment modalities. A $3 \%$ change in axial length (the $95 \%$ confidence limits of the formula) is towards the upper end of the magnitude of change seen in the dual focus lens study of Chamberlain et al. [7] over a three year period. As such, the predictions provided by the formula are too 'noisy' to be employed for precise tracking of myopic changes over time. In contrast, commercial biometers offer inter-observer or intraobserver repeatability ( $95 \%$ confidence limits) of $\pm 0.06 \mathrm{~mm}(\sim 0.25 \%)$ or better, $[26,27]$, indicative of a precise capability for tracking axial length change.

\section{Conclusion}

This work indicates that considering corneal curvature readings alongside refractive error measurement offers a good estimate of absolute axial length, and this estimate becomes less accurate if refractive error alone is used as a sole proxy for axial length. The formula developed provides extra clinical information to optometrists and opticians in the community (particularly those without access to dedicated biometry instrumentation) considering myopia management options for their patients and can be used in conjunction with published axial length risk parameters. However, practitioners wishing to precisely monitor change in axial length should utilise a commercial biometric device.

\section{Acknowledgement}

This manuscript was prepared with the support of an educational grant from CooperVision, Inc. The Misight(r) 1 day study referred to in this paper was funded by CooperVision, Inc. The NICER study was funded by the College of Optometrists.

\section{References}

[1] B.A. Holden, T.R. Fricke, D.A. Wilson, M. Jong, K.S. Naidoo, P. Sankaridurg, et al., Global Prevalence of Myopia and High Myopia and Temporal Trends from 2000 through 2050, Ophthalmology. 123 (2016) 1036-1042.

[2] Y.H. Guo, H.Y. Lin, L.L.K. Lin, C.Y. Cheng, Self-reported myopia in Taiwan: 2005 Taiwan National Health Interview Survey, Eye (Lond) 26 (2012) 684-689.

[3] S.K. Jung, J.H. Lee, H. Kakizaki, D. Jee, Prevalence of myopia and its association with body stature and educational level in 19-year-old male conscripts in Seoul, South Korea, Invest Ophthalmol Vis Sci 53 (2012) 5579-5583.

[4] C.W. McMonnies, Clinical prediction of the need for interventions for the control of myopia, Clin Exp Optom 98 (2015) 518-526.

[5] J.S. Wolffsohn, A. Calossi, P. Cho, K. Gifford, L. Jones, M. Li, et al., Global trends in myopia management attitudes and strategies in clinical practice, Cont Lens Anterior Eye 39 (2016) 106-116.

[6] A. Ruiz-Pomeda, B. Pérez-Sánchez, I. Valls, F.L. Prieto-Garrido, R. GutiérrezOrtega, C. Villa-Collar, MiSight Assessment Study Spain (MASS). A 2-year randomized clinical trial, Graefes Arch Clin Exp Ophthalmol 93 (2018) 336-11.

[7] P. Chamberlain, S.C. Peixoto-De-Matos, N.S. Logan, C. Ngo, D. Jones, G. Young, A 3-Year Randomized Clinical Trial of MiSight Lenses for Myopia Control, Optometry and Vision Science 96 (2019) 556-567.

[8] P. Cho, S.-W. Cheung, Retardation of Myopia in Orthokeratology (ROMIO) Study: A 2-Year Randomized Clinical TrialRetardation of Myopia in Orthokeratology Study, Invest Ophthalmol Vis Sci 53 (2012) 7077-7085.

[9] J. Santodomingo-Rubido, C. Villa-Collar, B. Gilmartin, R. Gutiérrez-Ortega, Myopia control with orthokeratology contact lenses in Spain: refractive and biometric 
changes, Invest Ophthalmol Vis Sci 53 (2012) 5060-5065.

[10] J.J. Walline, L.A. Jones, L.T. Sinnott, Corneal reshaping and myopia progression, British Journal of Ophthalmology 93 (2009) 1181-1185.

[11] J. Walline, M. Smith, Controlling myopia progression in children and adolescents, AHMT 6 (2015) 133-8.

[12] A. Chia, Q.-S. Lu, D. Tan, Five-Year Clinical Trial on Atropine for the Treatment of Myopia 2: Myopia Control with Atropine 0.01\% Eyedrops, Ophthalmology 123 (2016) 391-399.

[13] J. Vongphanit, P. Mitchell, J.J. Wang, Prevalence and progression of myopic retinopathy in an older population, Ophthalmology 109 (2002) 704-711.

[14] A. Ogawa, M. Tanaka, The relationship between refractive errors and retinal detachment--analysis of 1,166 retinal detachment cases, Jpn. J. Ophthalmol 32 (1988) 310-315.

[15] P. Mitchell, F. Hourihan, J. Sandbach, J. Jin Wang, The relationship between glaucoma and myopia, Ophthalmology 106 (1999) 2010-2015.

[16] R. Lim, P. Mitchell, R.G. Cumming, Refractive associations with cataract: the Blue Mountains Eye Study, Invest Ophthalmol Vis Sci 40 (1999) 3021-3026.

[17] D.I. Flitcroft, The complex interactions of retinal, optical and environmental factors in myopia aetiology, Progress in Retinal and Eye Research 31 (2012) 622-660.

[18] X. Cheng, N.A. Brennan, Y. Toubouti, M.A. Bullimore, Modelling of cumulative treatment efficacy in myopia progression interventions, Annual Meeting of the Association for Research in Vision and Opthalmology 2019 E-abstract 4345.

[19] J.W.L. Tideman, M.C.C. Snabel, M.S. Tedja, G.A. van Rijn, K.T. Wong, R.W.A.M. Kuijpers, et al., Association of Axial Length With Risk of Uncorrectable Visual Impairment for Europeans With Myopia, JAMA Ophthalmology 134 (2016) 1355.

[20] A. Ortiz, V. Galvis, A. Tello, V. Viaña, M.I. Corrales, M. Ochoa, et al., Comparison of three optical biometers: IOLMaster 500, Lenstar LS 900 and Aladdin, Int Ophthalmol 238 (2018) 765.

[21] O. Polat, Z. Baysal, S. Özcan, S. İnan, Ü.Ü. İnan, Comparison of Anterior Segment Measurements Obtained by Aladdin Optical Biometer and Sirius Corneal Topography, TJO 46 (2016) 259-263.

[22] J. Bland, D. Altman, Statistical methods for assessing agreement between two methods of clinical measurement, Lancet. 327 (1986) 307-310.

[23] S.J. McCullough, L. O'Donoghue, K.J. Saunders, Six Year Refractive Change among White Children and Young Adults: Evidence for Significant Increase in Myopia among White UK Children, PLoS ONE 11 (2016) e0146332.

[24] L. O'Donoghue, K.J. Saunders, J.F. McClelland, N.S. Logan, A.R. Rudnicka, B. Gilmartin, et al., Sampling and measurement methods for a study of childhood refractive error in a UK population, The British Journal of Ophthalmology 94 (2010) $1150-1154$.

[25] K.M.M. Breslin, L. O'Donoghue, K.J. Saunders, A prospective study of spherical refractive error and ocular components among Northern Irish schoolchildren (the NICER study), Invest Ophthalmol Vis Sci 54 (2013) 4843-4850.

[26] Y. Hua, W. Qiu, Q. Xiao, Q. Wu, Precision (repeatability and reproducibility) of ocular parameters obtained by the Tomey OA-2000 biometer compared to the IOLMaster in healthy eyes, PLoS ONE 13 (2018) e0193023.

[27] P. Mandal, E.J. Berrow, S.A. Naroo, J.S. Wolffsohn, D. Uthoff, D. Holland, et al., Validity and repeatability of the Aladdin ocular biometer., The British Journal of Ophthalmology 98 (2014) 256-258. 


\section{Figure legends}

245 Figure 1: Bland-Altman chart showing the relationship between the difference in axial length 246 (measured - calculated) versus mean axial length for the dataset of Chamberlain et al. [7] 247 The red line indicates the mean difference between the two methods and the dotted lines 248 show the 95\% limits of agreement as described by Bland and Altman (1986). [22]

Figure 2: Bland-Altman chart showing the relationship between the difference in axial length (measured - calculated) versus mean axial length for the NICER dataset [23] The red line indicates the mean difference between the two methods and the dotted lines show the $95 \%$ limits of agreement as described by Bland and Altman (1986). [22] 
254 Figure 1.

255

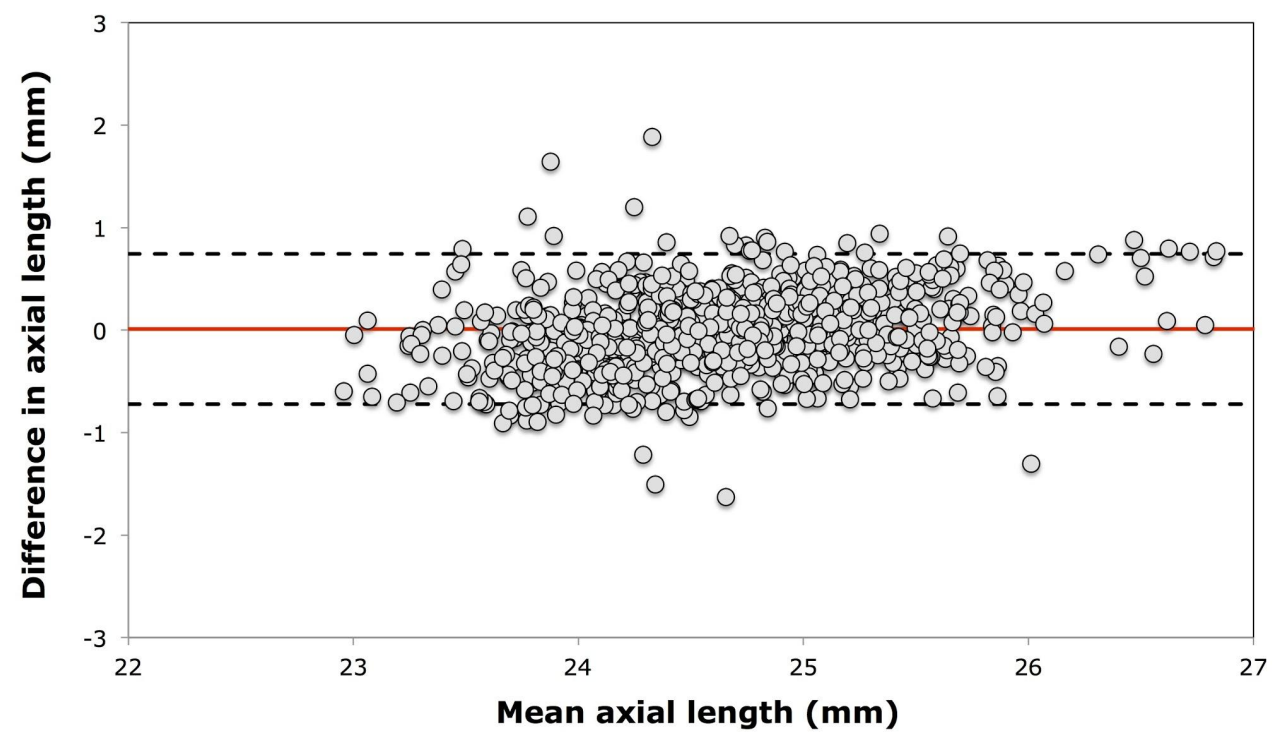

256

257

258 
259 Figure 2.

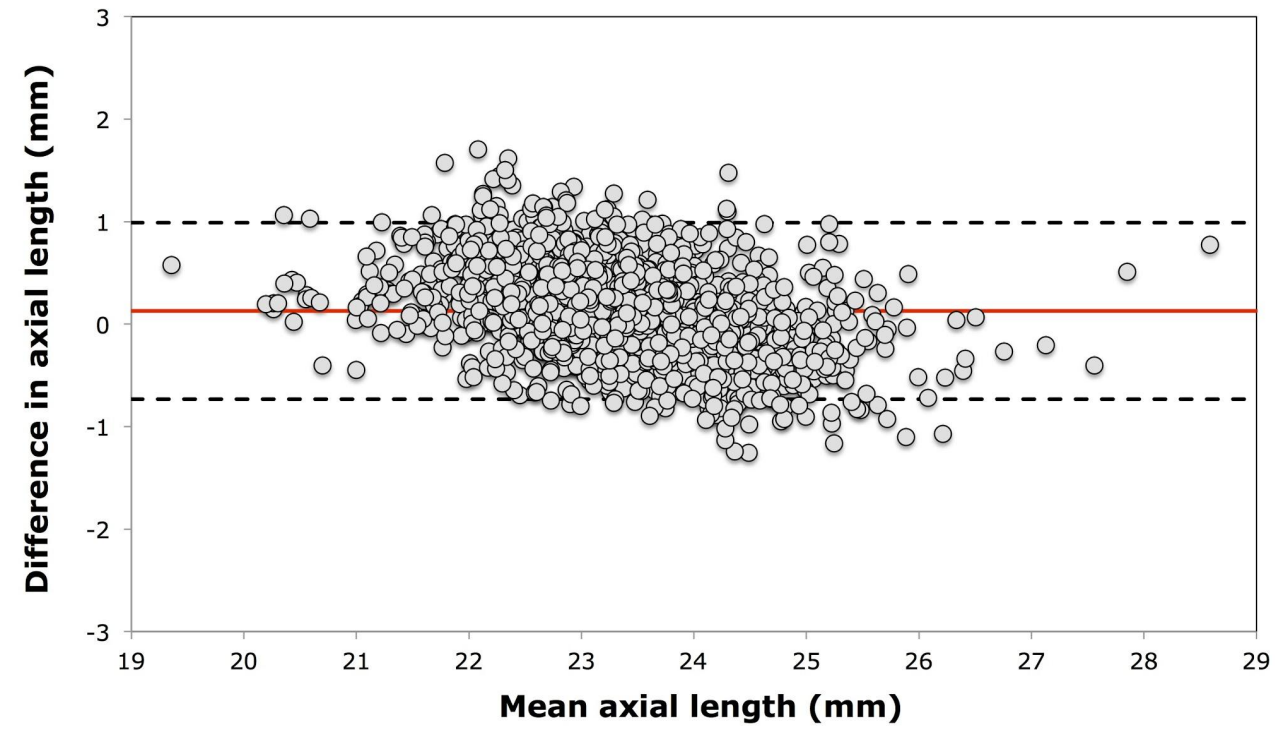




\section{Estimation of ocular axial length from conventional optometric measures}

Philip B. Morgan*

Sara J. McCullough^^

Kathryn J. Saunders^

* Eurolens Research, Division of Pharmacy and Optometry, University of Manchester, Dover Street, Manchester M13 9PL

^ Centre for Optometry and Vision Science Research, Ulster University, Cromore Road, Coleraine BT52 1SA

\section{Key words}

Axial length, topography, refraction, refractive error, myopia 